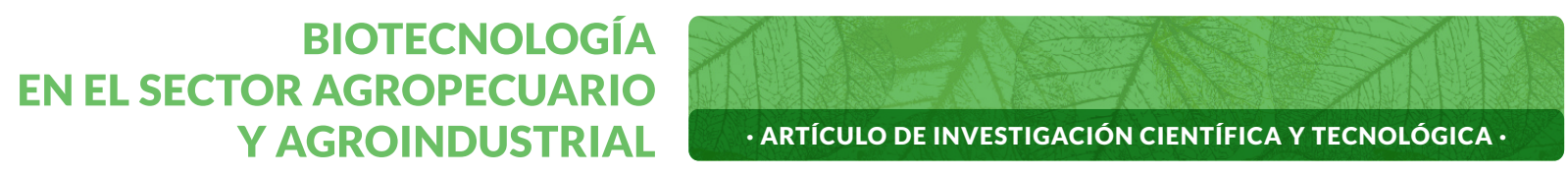

\title{
¿Cómo los consumidores valoran atributos de calidad de carne bovina y su disposición a pago?*
}

\section{How consumers assess beef quality cues and the willingness to purchase?}

\section{Como os consumidores valorizam atributos de qualidade da carne bovina e sua disposição a pagar?}

\author{
BARRAGÁN-HERNÁNDEZ, WILSON ${ }^{1}$; MAHECHA-LEDESMA, LILIANA²; \\ OLIVERA-ANGEL, MARTHA ${ }^{3}$; ANGULO-ARIZALA, JOAQUÍN ${ }^{4}$
}

Historial del Artículo

Recibido para evaluación: 23 de Abril 2020.

Aprobado para publicación: 20 de Octubre 2020

* $\quad$ Proyecto de investigación de origen: “Determinación de la calidad de carne bovina y la aceptación por parte del consumidor mediante el uso de pruebas con base en infrarrojo cercano". Financiación: Ministerio de Ciencia Tecnología e Innovación (MINCIENCIAS) en la convocatoria 727 de 2015, la estrategia de sostenibilidad de los grupos de investigación GRICA y Biogénesis de la Universidad de Antioquia y por la Corporación Colombiana de Investigación Agropecuaria (AGROSAVIA). Culminación:30 de junio de 2020.

1 Corporación Colombiana de Investigación Agropecuaria (AGROSAVIA). Centro de investigación Turipaná. MSc, Estudiante de doctorado en Ciencias Animales. Cereté, Colombia. https://orcid.org/0000-0003-3528-4296

2 Universidad de Antioquia, Facultad de Ciencias Agrarias, Grupo de Investigación en Ciencias Agrarias (GRICA). Dr. Agricultura y Medio Ambiente. Medellín, Colombia. https://orcid.org/0000-0003-3377-8399

3 Universidad de Antioquia, Facultad de Ciencias Agrarias, Grupo de investigación Biogénesis, Dr.Sci.Agr. Medellín, Colombia. https://orcid.org/0000-0001-7853-4406

4 Universidad de Antioquia, Facultad de Ciencias Agrarias, Grupo de Investigación en Ciencias Agrarias (GRICA). Dr. Ciencias Animales. Medellín, Colombia. https://orcid. org/0000-0003-3352-8795

Correspondencia: liliana.mahecha@udea.edu.co 


\section{RESUMEN}

La percepción de calidad de carne es un concepto complejo y requiere de análisis multivariados para entender su estructura y conceptualización. El objetivo del estudio fue valorar la percepción de calidad de carne por parte del consumidor y la disponibilidad a pago por información de calidad. Se aplicaron 400 encuestas bajo un cuestionario estructurado. La encuesta indagó sobre el perfil socioeconómico del consumidor y la percepción sobre características intrínsecas y extrínsecas de la carne, perfil de consumo por información nutricional, estilo de vida, preferencias de cortes y frecuencia de compra, y disposición a pago por información nutricional, empleando una escala Likert. Los datos se analizaron mediante modelos de ecuaciones estructurales (SEM) y pruebas $\mathrm{chi}^{2}$. El $S E M$ registró un ajuste satisfactorio ( $C F I=0,978, R M S E A=0,17)$. Los resultados indicaron que el consumidor conceptualiza $(P<0,05)$ la carne con base en aspectos intrínsecos (color, suavidad y pérdida de agua), extrínsecos (precio, bienestar animal y confianza) y la actitud de consumo saludable. El encuestado no consideró el concepto de calidad para la disposición a pago por información. Las mujeres, los consumidores entre 37-55 años y los empleados, registraron la puntuación más alta $(P<0,05)$ en el concepto de calidad de la carne.

\section{ABSTRACT}

Beef consumer's perception is complex and demands multivariate analyses to understand their structure and concept. This study aimed to evaluated beef consumer's perception and willingness to pay related to nutritional information. A total of 400 of structured surveys were applied. Surveyed were requested for information related to socioeconomic status and their perception about intrinsic and extrinsic beef cues, healthy consumption, nutritional information and willingness to pay, on a Likert scale. A structural equation model (SEM) and Chi-square were used to analyze. SEM showed a successful fit (CFI=0,978, RMSEA $=0,17)$. The beef consumer's perception was conceptualized as intrinsic (color, tenderness, and water holding capacity), extrinsic (price, animal welfare, and trusted), and healthy consumption. Women, consumers between 37 and 55 years old, and employees were consumers with a better beef quality concept.

\section{RESUMO}

A percepção do consumidor de carne bovina é complexa e exige análises multivariadas para entender sua estrutura e conceito. Este estudo teve como objetivo avaliar a percepção e disposição dos consumidores de pagar por informações nutricionais. Foram aplicados 400 inquéritos estruturados. A pesquisa investigou o perfil relacionado ao status socioeconômico e sua percepção so-

Cómo citar este artículo: BARRAGÁN-HERNÁNDEZ,WILSON; MAHECHA-LEDESMA, LILIANA; OLIVERA-ANGEL, MARTHA; ANGULO-ARIZALA, JOAQUÍN. ¿Cómo los consumidores valoran atributos de calidad de carne bovina y su disposición a pago?. Biotecnología en el sector agropecuario y agroindustrial, v. 19, n. 1, 2021, p. 167-179. Doi: https://doi.org/10.18684/BSAA(19)167-179

\section{PALABRAS CLAVES:}

Características cárnicas; Consumidores; Expectativas de consumo; Percepción de compra; Preferencias.

\section{KEYWORDS:}

Meat quality; Consumers; Consumption expectations; Purchase perception; Preference.

\section{PALAVRAS-CHAVE:}

Características da carne; Consumidor; Expectativas de consumo; Percepção de compra, Preferencias. 
bre as sinais intrínsecas e extrínsecas da carne bovina, consumo saudável, informações nutricionais e disposição para pagar, em escala Likert. Um modelo de equação estrutural (SEM) e qui-quadrado foram utilizados para o analise. O SEM mostrou um ajuste satisfatório $(C F I=0,978, R M S E A=0,17)$. A percepção do consumidor de carne bovina foi conceituada como consumo intrínseco (cor, maciez e capacidade de retenção de água), extrínseco (preço, bem-estar animal e confiável) e na atitude de consumo saudável. Mulheres, consumidores entre 37 e 55 anos e funcionarios, registraram a maior pontuação $(P<0,05)$ no conceito de qualidade da carne.

\section{INTRODUCCIÓN}

La calidad de la carne, percibida por el consumidor, se define como un concepto complejo [1], construido sobre la base de atributos intrínsecos y extrínsecos de la carne, experiencias previas y expectativas de consumo [2,3]. Los atributos intrínsecos, se han descrito como características inherentes a la carne, incapaces de ser modificadas sin alterar substancialmente la naturaleza del producto. Por su parte, los atributos extrínsecos están relacionados con el producto, pero no son inherentes a su naturaleza, como el precio, la marca, el sistema productivo entre otros $[1,4]$

Recientemente, varios investigadores se han preocupado por entender la percepción del consumidor de carne bovina [5,6] en conceptos tan generales como el producto mismo [7] específicos como la percepción de inocuidad [8], sostenibilidad [6], palatabilidad y marca comercial [9] o preferencia en contenido nutricional [10]. Este esfuerzo se relaciona con la necesidad de generar información que le permita a la industria de carne bovina, ofrecer un producto con mayor probabilidad de ser aceptado, con el objeto de enfrentar la disminución per capita del consumo de carne [5].

Ngapo et al. [11] han señalado que los consumidores pueden utilizar más de dos atributos de la carne para construir su concepto de calidad, entre ellos, los atributos intrínsecos como el color y el contenido de grasa, son importantes. Por su parte Flowers et al. [10], señalaron que el precio es el atributo de la carne que determina la elección del producto en consumidores americanos; sin embargo, obviando el precio, estos consumidores apuntan la experiencia de consumo y el grado USDA (United State Department of Agricultural) de la carne como los atributos más importantes.

El entendimiento de la percepción del consumidor se considera una herramienta necesaria para dinamizar la industria cárnica [12], toda vez que los atributos de la carne influyen en el concepto de calidad del producto, en la atención y frecuencia de elección que expresa el consumidor al momento de la compra y en la disponibilidad de pago por calidad $[7,13,14]$

En Colombia, el consumo per capita de carne bovina disminuyó de 20 kg.año-1 en el año 2011, a 18,6 kg.año ${ }^{-1}$ en 2019. Por su parte, sustitutos proteicos que compiten por su bajo costo como el pollo y el cerdo, aumentaron en 11,8 kg y 5,58 kg, al incrementar su consumo per capita de 23,8 kg.año-1 a 35,6 kg.año-1 y de 5,52 kg.año-1 a 11,1 kg.año-1 ${ }^{-1}$ respectivamente. Asimismo, el pescado, considerado un sustituto proteico no tan económico, presentó un incremento de $4,2 \mathrm{~kg}$ (8,7 kg.año-1 vs 4,52 kg.año-1), en el mismo período [15]. Según Camacho et al. [16], las restricciones al consumo de carne en el consumidor colombiano, pueden estar asociadas a la percepción sobre potenciales problemas de salud derivados de su consumo.

Para el caso Colombiano, son escasas las investigaciones enfocadas a entender la percepción o caracterización del consumidor de carne bovina, pese a ser una de las demandas de la cadena cárnica [17]. FEDEGAN [18] desarrolló una investigación para caracterizar el consumidor de carne bovina con resultados que dan orientación sobre preferencias y características de la carne relacionadas con la calidad. Sin embargo, la percepción del consumidor no se puede entender con base en una sola característica, puesto que es un concepto complejo con factores interrelacionados, por lo cual, la aplicación de herramientas de análisis multivariado permitiría identificar los factores de la carne y sus interrelaciones que influyen en la percepción del consumidor. Por consiguiente, el objetivo fue determinar la percepción del consumidor sobre la calidad de carne bovina aplicando un modelo estructural y su relación con la disposición a pago por calidad. 


\section{MÉTODO}

La encuesta se desarrolló para abordar los principales aspectos relacionados con la percepción de la calidad de la carne bovina. Las bases teóricas para el desarrollo de las preguntas fueron revisadas previamente por Henchion et al. [1], Grunert et al. [2], Font I Furnols et al. [3] y Frank et al. [19].

El formato para la captura de información abordó aspectos relacionados con el perfil socioeconómico del encuestado, frecuencias y restricciones en el consumo de carne bovina, percepción relacionada con características intrínsecas y extrínsecas del producto, aspectos orientados a investigar el perfil de consumo por información nutricional y estilo de vida, preferencias de cortes y frecuencia de compra, y disposición a pago por información nutricional, enfocada a perfil de ácidos grasos.

Las preguntas relacionadas con el perfil socioeconómico registraron los principales niveles en las categorías: género, rango salarial, nivel de estudios, ocupación y estado civil, así como también, la edad en años. Con relación a la percepción, el cuestionario aplicado (30 preguntas) empleó una escala tipo Likert de cinco puntos, donde el número 1 se relacionó con la percepción "Me disgusta extremadamente" y el 5 con "Me gusta extremadamente". La disposición a pago y el valor en pesos colombianos (\$COP) por información nutricional, se indagaron en una pregunta dicotómica $(\mathrm{Si}, \mathrm{No})$ y en el valor monetario que el consumidor informó que estaba dispuesto a pagar sobre el precio medio de la carne que compraba, respectivamente. Las preguntas y el modelo estructural aplicado en esta investigación, fueron validados mediante encuestas vía email en 602 participantes, con una tasa de respuesta del 18\% [20].

La investigación se llevó a cabo en octubre de 2019 en municipios del área Metropolitana de Antioquia (Colombia) en los $75^{\circ} 33^{\prime} 48,92^{\prime \prime}$ longitud Oeste y los 6 $6^{\circ} 15^{\prime} 6.62^{\prime \prime}$ de latitud Norte, con una altitud media de 1.495 m.s.n.m. y temperatura promedio de $25^{\circ} \mathrm{C}$. La muestra se estimó con base en los datos poblacionales informados por Mejía et al. [21], aplicando un muestreo poblacional estratificado por condición socioeconómica [14,22]. El cuestionario fue evaluado en un total de 400 consumidores de carne, asociados a una cadena comercial de supermercado y se aplicó en días no consecutivos, con el objeto de maximizar la representación de los consumidores [23]. Así mismo, se seleccionó el área de venta de carnes para la aplicación de la encuesta, con el objeto de garantizar que el encuestado fuera consumidor de este producto [14]. Cada encuestado fue indagado por su participación mediante un apartado de consentimiento informado en el formulario y su contribución fue compensada con un subproducto cárnico.

El modelo estructural propuesto consideró cuatro dimensiones exógenas (constructos formados a partir de variables observadas) relacionadas con la percepción de atributos intrínsecos de la carne, percepción extrínseca, actitud frente al consumo saludable y expectativa por información nutricional y una endógena (constructos formados a partir de variables latentes), asociada a la percepción de calidad de la carne. En cada uno de los constructos exógenos (cuadro 1), se validaron los criterios de adecuación muestreal (MSA) de Kaiser-Meyer-Olkin validando la pertinencia de las variables con un valor >0,5, y Alpha de Cronbach con índice >0,6 [24], empleando la librería psych del software R-Project [25].

Cuadro 1. Validación de variables latentes exógenas e hipótesis en el modelo estructural.

\begin{tabular}{|c|c|c|c|c|}
\hline $\begin{array}{l}\text { Latente } \\
\text { endógena }\end{array}$ & $\begin{array}{l}\text { Latente } \\
\text { exógena }\end{array}$ & Hipótesis & $\begin{array}{l}\text { Alpha de } \\
\text { Cronbrach }\end{array}$ & $\mathrm{MSA}^{1}$ \\
\hline \multirow{4}{*}{$\begin{array}{l}\text { Percepción de } \\
\text { calidad de carne }\end{array}$} & $\begin{array}{l}\text { Percepción de atributos } \\
\text { intrínseco }\end{array}$ & $\begin{array}{l}\text { H1. Los consumidores valoran los atributos intrínsecos } \\
\text { como parte del concepto de calidad }\end{array}$ & 0,603 & 0,71 \\
\hline & $\begin{array}{l}\text { Percepción de atributos } \\
\text { extrínsecos }\end{array}$ & $\begin{array}{l}\text { H2. Los consumidores valoran los atributos extrínsecos } \\
\text { como parte del concepto de calidad }\end{array}$ & 0,606 & 0,64 \\
\hline & $\begin{array}{l}\text { Actitud frente } \\
\text { al consumo saludable }\end{array}$ & $\begin{array}{l}\text { H3. La actitud frente a un consumo saludable está } \\
\text { relacionada con la percepción de calidad de carne }\end{array}$ & 0,756 & 0,75 \\
\hline & $\begin{array}{l}\text { Expectativa por información } \\
\text { de calidad }\end{array}$ & $\begin{array}{l}\text { H4. La expectativa de información nutricional } \\
\text { en la carne forma parte del concepto de calidad }\end{array}$ & 0,651 & 0,75 \\
\hline
\end{tabular}

${ }^{1}$ Criterio de adecuación muestral de Kaiser-Meyer-Olkin 
El análisis de ecuaciones estructurales se realizó empleando la librería lavaan del software R-Project [26]. La fiabilidad del análisis se consideró con base en la ausencia de varianzas negativas en el error y cargas factoriales estandarizadas > 1. Así mismo, se evaluaron los criterios de bondad y ajuste relacionados con el cuadrado medio estandarizado del residual (SRMR por sus siglas en inglés) y el cuadrado medio del error por aproximación (RMSEA, por sus siglas en inglés), con un límite de aceptación < 0,08, y los índices de ajuste comparativo CFI (Comparative Fit Index) y TLI (Tuker-Lewis Index), con valores de aceptación >0,9 [24,27]. Bajo el modelo seleccionado, se realizó la predicción de las variables latentes evaluadas, en la escala Likert de cinco puntos.

El contraste de las variables latentes generadas en el modelo estructural, con las variables de tipificación del consumidor, se realizó aplicando una prueba de $\mathrm{Chi}^{2}$, rechazando la hipótesis de independencia con un valor alpha >0,05.

\section{RESULTADOS}

El cuadro 2 presenta los resultados del perfil socioeconómico y demográfico de los consumidores encuestados. Los resultados sugieren que la participación de hombres y mujeres mostró una representación similar a la reportada en reciente censo para la población colombiana ( $48 \%$ mujeres y $51 \%$ hombres), al igual que la proporción de personas que se dedican a las actividades del hogar (19\%) y de empleados (47\%), pero diferente al porcentaje de estudiantes (18\%) y de pensionados (3,6\%) [28].

Cuadro 2. Características socioeconómicas y demográficas de los consumidores de carne (n=400).

\begin{tabular}{|c|c|c|}
\hline Variable & Nivel & Porcentaje \\
\hline \multirow[t]{2}{*}{ Género } & Masculino & $41,32 \%$ \\
\hline & Femenino & $58,67 \%$ \\
\hline \multirow[t]{4}{*}{ Edad (Años) } & $<37$ & $31,78 \%$ \\
\hline & $37-55$ & $38,14 \%$ \\
\hline & $55-74$ & $28,11 \%$ \\
\hline & $>74$ & $1,95 \%$ \\
\hline Ingresos & No declara & $16,38 \%$ \\
\hline \multirow[t]{5}{*}{ (Número de SMLMV ${ }^{1}$ ) } & $<1$ & $11,49 \%$ \\
\hline & $1-2$ & $41,07 \%$ \\
\hline & $3-4$ & $21,02 \%$ \\
\hline & $5-6$ & $7,33 \%$ \\
\hline & $>6$ & $2,68 \%$ \\
\hline \multirow[t]{6}{*}{ Estado civil } & Casado(a) & $43,27 \%$ \\
\hline & Separado(a) & $6,35 \%$ \\
\hline & Soltero(a) & $30,56 \%$ \\
\hline & Viudo(a) & $3,66 \%$ \\
\hline & Unión libre & $15,64 \%$ \\
\hline & Otro & $0,4 \%$ \\
\hline \multirow[t]{6}{*}{ Nivel educativo } & Primaria & $8,55 \%$ \\
\hline & Secundaría & $35,94 \%$ \\
\hline & Tecnología & $21,51 \%$ \\
\hline & Profesional & $22,00 \%$ \\
\hline & Posgrado & $11,00 \%$ \\
\hline & Otro & $0,90 \%$ \\
\hline \multirow[t]{6}{*}{ Ocupación } & Servicio al hogar & $19,55 \%$ \\
\hline & Desempleado & $0,7 \%$ \\
\hline & Empleado & $40,58 \%$ \\
\hline & Pensionado & $11,49 \%$ \\
\hline & Estudiante & $4,15 \%$ \\
\hline & Independiente & $23,47 \%$ \\
\hline
\end{tabular}

${ }^{1}$ salario mínimo legal mensual vigente 
EI SEM fue validado de acuerdo a Bagozzi and Yi [29] y se aceptó la hipótesis de igualdad entre las covarianzas calculadas y predichas $\left(X^{2}{ }_{d f 381} P>0,05\right)$ con ajuste satisfactorio ( $C F I=0,978, T L I=0,973, R M S E A=0,17$ y $\left.S R M R=0,48\right)$. La construcción de las variables latentes de orden exógenas y endógenas, se presentan en el cuadro 3.

\section{Percepción intrínseca y extrínseca de la carne}

Los consumidores valoran $(\mathrm{P}<0,05)$ principalmente la calidad intrínseca de la carne a través de la percepción del color, el concepto de suavidad y la valoración de la pérdida de agua. Por el contrario, la conceptualización de la maduración de la carne no es considerada $(C F=0,10, P>0,05)$.

Las características intrínsecas valoradas por los consumidores en el presente estudio, están en concordancia con los atributos intrínsecos de la carne informados por consumidores italianos [30], americanos [31] y mexicanos [11]. Entre estos atributos, el color de la carne y la pérdida de agua han sido informados como las primeras características que usa el consumidor para valorar la frescura del producto [1] y la terneza o suavidad, como uno de los factores más determinantes en la elección de la carne [32].

Con relación a la grasa como atributo intrínseco, la percepción sobre la influencia en la mejora de la calidad de la carne, aunque presentó un efecto significativo dentro de la conceptualización intrínseca, obtuvo una baja carga factorial ( $C F=0,208$, cuadro 3). Estos resultados son opuestos a lo informado por Henchion et al. [1] y Font-i-Furnols and Guerrero [3], quienes señalan que la grasa visible es una de las señales de calidad de carne con mayor influencia en la percepción del consumidor. Así mismo, los resultados sobre la percepción de la grasa plantean la falta de conocimiento del consumidor encuestado sobre su efecto en la mejora de la experiencia de consumo [5], opuesto a lo reportado por Ngapo et al. [11], quienes demostraron que el consumidor mexicano, aunque presenta una percepción negativa de la grasa en la carne, reconoce que debe existir un mínimo de grasa para mejorar su sabor.

En el entorno colombiano, el concepto de maduración de la carne es poco conocido, como también fue demostrado por FEDEGAN [18] y Barragán et al. [20]. Sin embargo, está bien documentado el efecto positivo de los cambios bioquímicos y estructurales de la maduración en la palatabilidad (terneza, jugosidad y sabor) de la carne [33]. Por lo cual, los resultados del presente estudio permiten plantear la pobre educación en calidad de carne del consumidor colombiano, similar al caso del consumidor venezolano descrito por Arenas-De Moreno et al. [32], quienes indican que el concepto de maduración tiene mayor apropiación entre los consumidores de la región central de Venezuela (mayor preferencia y educación en calidad de carne) comparado con el consumidor de la región occidental.

En la percepción extrínseca, el concepto de bienestar animal, el sitio de compra de la carne y el precio, resaltan entre los atributos que más contribuyen a la formación del concepto $(P<0,05)$. Estos resultados concuerdan con Miranda de Lama et al. [34] quienes describieron la relación entre el bienestar animal y el consumidor de carne mexicano, y con Merlino et al. [30], que agruparon consumidores de carne ( $n=401)$ en cinco grupos, el primero y más importante (32,5\% de los consumidores), motivado por el precio de la carne y el cuarto (12,9\%) caracterizados por la importancia en el bienestar animal.

El precio ha sido uno de los atributos de la carne de mayor impacto en la percepción del consumidor [2,7,10], sin embargo, a diferencia de reportes $[10,30]$ en los cuales los consumidores priorizan el precio sobre otros atributos de la carne, resalta para este estudio la participación de la confianza del empacado como atributo de mayor carga factorial en la formación del concepto de calidad extrínseca.

La reorganización de importancia de los atributos en el concepto de calidad de carne fue informada por Merlino et al. [30], quienes indican que los consumidores modifican la jerarquía de los atributos que evalúan según el lugar donde compran la carne, lo cual pudo influenciar que los consumidores del presente estudio experimentaran una modificación de prioridades en lo atributos extrínsecos, dado que el experimento fue desarrollado en el área de venta de carne de un supermercado. 
Cuadro 3. Variables latentes y cargas factoriales en el modelo de ecuaciones estructurales para la percepción de la calidad de la carne bovina.

\begin{tabular}{|c|c|c|c|}
\hline Latente & Variable observada & CF & Valor $\mathrm{p}$ \\
\hline \multirow{10}{*}{$\begin{array}{l}\text { Percepción } \\
\text { intrínseca }\end{array}$} & La suavidad de la carne es fundamental cuando voy a comprar & 0,420 & $>0,001$ \\
\hline & Si la carne escurre agua, no la compro & 0,354 & $>0,001$ \\
\hline & Reviso el color cuando compro la carne res & 0,505 & $>0,001$ \\
\hline & Prefiero carne con color rojo oscuro sobre un color rojo brillante & 0,173 & 0,006 \\
\hline & Prefiero consumir carne fresca sobre carne madurada & 0,102 & 0,096 \\
\hline & Considero que la grasa en la carne mejora su sabor & 0,208 & 0,001 \\
\hline & Me gusta la carne de res sobre otras carnes & 0,274 & $>0,001$ \\
\hline & Confío más en comer carne de res que de pollo o cerdo & 0,236 & $>0,001$ \\
\hline & Confío más en comer carne de res que de pescado & 0,183 & 0,004 \\
\hline & La forma de cocinar determina el tipo de carne que compro & 0,267 & $>0,001$ \\
\hline \multirow{6}{*}{$\begin{array}{l}\text { Percepción } \\
\text { extrínseca }\end{array}$} & Quisiera comer más carne de res pero el precio me limita & 0,169 & $>0,001$ \\
\hline & La forma como está empacada la carne me genera confianza & 0,501 & 0,015 \\
\hline & Conozco el proceso de beneficio y me interesa el bienestar animal & 0,299 & 0,003 \\
\hline & Considero que la carne es costosa y por eso no como todos los días & 0,422 & 0,016 \\
\hline & Usualmente compro la carne en el mismo sitio & 0,227 & 0,038 \\
\hline & La información hacia el consumo de carne roja es negativa & $-0,167$ & 0,348 \\
\hline \multirow{5}{*}{$\begin{array}{l}\text { Expectativa } \\
\text { hacia información } \\
\text { nutricional }\end{array}$} & Reviso si el alimento contiene ácidos grasos omega y colesterol & 0,647 & $>0,001$ \\
\hline & Si la carne declarara el contenido de omega 3 o 6 me gustaría más & 0,730 & $>0,001$ \\
\hline & Si conociera cuánta proteína aporta la carne que como sería mejor & 0,592 & $>0,001$ \\
\hline & Limito mi consumo de res por lo que dicen sobre la grasa & 0,350 & $>0,001$ \\
\hline & Con relación a la grasa prefiero carne de res magra (poca grasa) & 0,314 & $>0,001$ \\
\hline \multirow{6}{*}{$\begin{array}{l}\text { Consumo } \\
\text { saludable }\end{array}$} & Me preocupo por comer dietas saludables & 0,517 & $>0,001$ \\
\hline & Compro alimentos que sean lo más saludables para mí y mi familia & 0,632 & $>0,001$ \\
\hline & Cuando compro un alimento reviso su información nutricional & 0,893 & $>0,001$ \\
\hline & Me importa el contenido de grasa y proteína en los alimentos & 0,727 & $>0,001$ \\
\hline & Expectativa hacia información nutricional & 0,684 & $>0,001$ \\
\hline & Ddisposición a pago por información nutricional & 0,461 & $>0,001$ \\
\hline \multirow{11}{*}{$\begin{array}{l}\text { Percepción de la } \\
\text { calidad de la carne } \\
\text { bovina }\end{array}$} & Percepción intrínseca & 0,892 & $>0,001$ \\
\hline & Percepción extrínseca & 0,752 & 0,017 \\
\hline & Consumo Saludable & 0,711 & $>0,001$ \\
\hline & Con relación a la grasa prefiero carne de res magra (poca grasa) & 0,183 & 0,012 \\
\hline & El olor de la carne es importante a la hora de decidir su compra & 0,537 & $>0,001$ \\
\hline & Cuando compro un alimento reviso su información nutricional & $-0,364$ & 0,004 \\
\hline & Cuando compro carne me fijo en su contenido de grasa & 0,355 & $>0,001$ \\
\hline & Disposición a pago por información nutricional & $-0,249$ & 0,016 \\
\hline & La forma como está empacada la carne me genera confianza & 0,511 & 0,002 \\
\hline & Considero que la carne de res es una buena fuente de nutrientes. & 0,608 & $>0,001$ \\
\hline & Expectativa hacia información nutricional & 0,003 & 0,974 \\
\hline
\end{tabular}

$\mathrm{CF}=$ Carga Factorial

Contrario los reportes de Enciso et al. [14], FEDEGAN [18] y Barragán et al. [20], quienes exploraron la valoración de aspectos de calidad de carne en consumidores colombianos, este estudio identificó que el concepto de bienestar animal formó parte de la conceptualización de la calidad extrínseca de la carne bovina. Estos resultados permitirían explorar la posibilidad de sellos y/o etiquetas que aporten valor agregado a la carne, basado en 
experiencias previas que han demostrado que los consumidores podrían tener una disposición a pago por carne producida bajo esquemas de bienestar animal [34].

\section{Actitud frente al consumo saludable y percepción sobre información nutricional}

La expectativa de los consumidores asociada a la información nutricional de la carne, con enfoque en ácidos grasos estuvo conformada $(P<0,05)$ por la valoración positiva de conocer el contenido de ácidos grasos, la importancia de revisar la información nutricional asociada a los ácidos grasos y por la expectativa de apreciar más la carne si el consumidor conociera el contenido proteico. Así mismo, el constructo de expectativa por información, influyó positivamente $(P<0,05)$ la conceptualización de un hábito de consumo saludable, al igual $(P<0,05)$ que las variables observadas asociadas a la rutina de revisión de información nutricional en los alimentos y la importancia que el consumidor le da a los contenidos de grasa y proteína en los alimentos.

Lusk [35] afirmó que el 52,1\% de los consumidores americanos $(n=1.290)$ basa su criterio de alimento saludable en un único componente nutricional del alimento, entre los cuales el contenido de grasa y proteína ocupan el tercer y noveno puesto en orden de importancia, respectivamente. Con relación a nuestros resultados, el entendimiento de la percepción de la grasa y/o de sus componentes es un aspecto importante, dado influencia negativa de la información asociada a la grasa y su efecto en la salud de los consumidores de carne [36]. Al respecto, Flowers et al. [31] reportaron que los consumidores de carne desconocen y confunden los conceptos asociados a grasa y perfil de ácidos grasos (monoinsaturado, polinsaturada y grasa trans de origen ruminal) y que la pedagogía sobre el beneficio de estos nutrientes, impacta positivamente la decisión de compra.

\section{Concepto de calidad de carne bovina}

La variable latente de construcción endógena asociada a la conceptualización de calidad de la carne se describe en cuadro 3. El SEM validó $(P<0,05)$ todas las hipótesis planteadas, con excepción $(P>0,05)$ de la asociada a la expectativa de la información. Adicionalmente, las variables observadas relacionadas con la preferencia por carne magra, el olor, revisión del contenido de grasa y contenido nutricional, disposición a pago por información de calidad, el empaque y la preferencia de la carne como alimento nutritivo, influyeron $(P<0,05)$ en la construcción de la percepción de calidad.

La percepción de los atributos intrínsecos y extrínsecos de la carne, y la actitud hacia un consumo saludable representaron los conceptos de mayor contribución positiva a la formación de la percepción de calidad (CF > $0,70)$. Por el contrario, la revisión del contenido nutricional $(C F=-0,36)$ y la disposición a pago por información de calidad $(C F=-0,25)$, contribuyeron negativamente a la percepción de calidad de la carne. Estos resultados sugieren que el consumidor de carne se preocupa por un consumo saludable y estaría dispuesto a pagar más por información de calidad nutricional en otros alimentos, pero su percepción y confianza en la carne bovina sobrepasa la necesidad de conocer la información nutricional y desincentiva el pago por esta información.

Al igual que en este estudio, varios autores han demostrado la importancia de las características intrínsecas y extrínsecas de la carne en el concepto de calidad de carne [1,11,32]. Sin embargo, contrario al registrado en este trabajo, la literatura reporta casos exitosos de disposición a pago por información nutricional en la carne bovina [10,31], influenciados por la necesidad creciente del consumidor por compra con base en decisiones informadas [35]. Adicionalmente, Barragán et al. [20] mediante la aplicación por formulario electrónico de la encuesta usada en el presente estudio, encontraron una disposición positiva al pago por información nutricional en la carne bovina, probablemente asociado al muestreo por conveniencia $(n=108)$ que influyó en una muestra con mayor proporción de encuestados con formación universitaria y con grado de maestría (82\%) e ingresos económicos superiores a 5 SMLMV (38\%). 


\section{Relación entre tipificación de consumidores y la percepción de calidad de carne}

El análisis de dependencia entre las características del consumidor de carne y la predicción de la variable latente asociada a la percepción de la calidad de carne bovina identificada en el SEM, rechazó la hipótesis $(P<0,05)$ de independencia en las variables género, frecuencia en el consumo de carne y edad. Un mayor porcentaje de mujeres estuvo asociado a una puntuación más alta en la variable latente. Así como también, encuestados con una frecuencia de consumo de carne de $>4$ veces por semana y con edades entre los 37 y 55 años (cuadro 4).

La relación de dependencia entre las mujeres y la mayor calificación en la percepción de calidad de carne bovina pudo estar influenciada por factores asociados a los conceptos de alimentación saludable y percepción relacio-

Cuadro 4. Dependencia $\left(X^{2}\right)$ entre el porcentaje de respuestas asociadas a la tipificación del consumidor de carne y la predicción de la variable latente percepción de la calidad de carne bovina. ${ }^{1}$

\begin{tabular}{|c|c|c|c|c|c|}
\hline & & \multicolumn{3}{|c|}{ Predicción de variable latente $^{2}$} & \multirow[b]{2}{*}{ Valor de $\mathrm{P}$} \\
\hline & & 2 & 3 & 4 & \\
\hline \multirow{2}{*}{ Género } & Masculino & 0,00 & 15,86 & 23,53 & \multirow{2}{*}{0,0440} \\
\hline & Femenino & 0,26 & 17,65 & 42,71 & \\
\hline \multirow{6}{*}{$\begin{array}{l}\text { Ingresos } \\
(\mathrm{SMLMV})^{3}\end{array}$} & No declara & 0,26 & 7,42 & 9,46 & \multirow{6}{*}{0,2889} \\
\hline & $<1$ & 0,00 & 4,86 & 6,14 & \\
\hline & $1-2$ & 0,00 & 13,04 & 28,39 & \\
\hline & $3-4$ & 0,00 & 7,93 & 12,79 & \\
\hline & $5-6$ & 0,00 & 1,79 & 5,12 & \\
\hline & $>6$ & 0,00 & 1,28 & 1,53 & \\
\hline \multirow{6}{*}{ Nivel educativo } & Primaria & 0,00 & 2,30 & 6,65 & \multirow{6}{*}{0,0910} \\
\hline & Secundaría & 0,26 & 14,07 & 21,48 & \\
\hline & Tecnología & 0,00 & 6,39 & 14,83 & \\
\hline & Profesional & 0,00 & 9,72 & 13,04 & \\
\hline & Posgrado & 0,00 & 3,58 & 6,91 & \\
\hline & Otro & 0,00 & 0,26 & 0,51 & \\
\hline \multirow{6}{*}{ Ocupación } & Servicio al hogar & 0,26 & 6,39 & 13,30 & \multirow{6}{*}{0,0860} \\
\hline & Desempleado & 0,00 & 0,77 & 0,00 & \\
\hline & Empleado & 0,00 & 16,11 & 25,32 & \\
\hline & Pensionado & 0,00 & 2,81 & 8,18 & \\
\hline & Estudiante & 0,00 & 2,56 & 1,28 & \\
\hline & Independiente & 0,00 & 7,67 & 15,35 & \\
\hline \multirow{3}{*}{$\begin{array}{l}\text { Consumo de carne } \\
\text { (número de veces por } \\
\text { semana) }\end{array}$} & $1-2$ & 0,26 & 16,37 & 21,99 & \multirow{3}{*}{0,0290} \\
\hline & $2-3$ & 0,00 & 11,00 & 19,18 & \\
\hline & $>4$ & 0,00 & 8,95 & 22,25 & \\
\hline \multirow{2}{*}{ Restricción consumo } & $\mathrm{Si}$ & 0,26 & 7,93 & 9,46 & \multirow{2}{*}{0,6642} \\
\hline & No & 0,00 & 28,39 & 53,96 & \\
\hline \multirow{5}{*}{$\begin{array}{l}\text { Edad } \\
\text { (Años) }\end{array}$} & $<25$ & 0,00 & 7,16 & 4,60 & \multirow{5}{*}{0,0005} \\
\hline & $25-37$ & 0,00 & 8,70 & 11,76 & \\
\hline & $37-55$ & 0,26 & 9,72 & 27,62 & \\
\hline & $55-74$ & 0,00 & 9,97 & 18,16 & \\
\hline & $>74$ & 0,00 & 0,77 & 1,28 & \\
\hline
\end{tabular}

${ }^{1}$ Números resaltados en negrita representan el mayor porcentaje de respuestas entre las dos variables analizadas.

${ }^{2}$ Predicción de la variable latente de acuerdo con el modelo SEM donde 1 se asocia a menor puntuación de la percepción y 5 a mayor puntuación.

${ }^{3}$ Número de salario mínimo mensual legal vigente. 
nada con el contenido de grasa como fue demostrado en el modelo SEM del presente estudio. Lusk [35], reportó que las mujeres poseen mayor afinidad por el concepto de alimento saludable comparado con los hombres, describiendo este concepto, entre otros aspectos, como alimentos bajos en grasa. De igual forma, Banović et al. [13] concluyó que las mujeres son capaces de decidir de forma más rápida la elección de la carne bovina con bajo contenido de grasa, comparado con los hombres. Según los anteriores autores, esta elección se basa en la conceptualización de que alimentos bajos en grasa son más saludables.

La relación entre la mejor conceptualización de la calidad de la carne bovina y los consumidores de mayor frecuencia de carne por semana, puede estar asociada a la preferencia y confianza en el consumo de carne. Estos resultados presentan similitud con los reportes de Flowers et al. [10] y Arenas-De Moreno et al. [32], quienes relacionan una mayor frecuencia de consumo con mayor preferencia por la carne.

Por último, se registró una mayor apropiación del concepto de calidad de carne entre consumidores de 37 y 55 años. Estos resultados podrían estar asociados a ser un rango de edad productiva y menor influencia del precio de la carne en su adquisición como se pudo observar en la tendencia $(P=0,0860)$ de un mejor concepto de calidad de carne entre los consumidores con empleo. Realini et al. [37] identificó que los consumidores españoles de entre 41 y 60 años presentan mayor preferencia por la carne bovina comparado por consumidores jóvenes, quienes expresan restricción al consumo de carne por el precio y poseen menor preferencia por la carne por su estilo de vida.

\section{CONCLUSIONES}

La percepción de calidad de carne bovina de los consumidores encuestados se basó en la valoración de los factores intrínsecos y extrínsecos de la carne, en la actitud frente al consumo saludable y en variables relacionadas al producto, como el contenido de grasa y la preferencia de consumo, sin considerar importante la información nutricional. Estos resultados, aunque son válidos para el área de influencia del presente estudio, demuestran la adaptación de una metodología de evaluación de percepción del consumidor de carne bovina que puede ser aplicada en otras regiones de Colombia.

Conceptos reconocidos en el entorno científico que se asocian a mayor aceptación en la carne bovina, como es el caso de la maduración de la carne, no son valorados por el consumidor. Esta situación evidencia la necesidad de la industria de carne bovina de educar al consumidor sobre calidad de carne.

La percepción de calidad de carne bovina en los consumidores encuestados, no consideró la posibilidad de pagar más dinero por información de calidad. La confianza y la preferencia en la carne subvaloran la información nutricional. Así mismo, se identificó que los consumidores desconocen o no valoran el perfil de ácidos grasos y las implicaciones en la nutrición y salud. En general, el concepto de grasa se asocia a una percepción negativa, lo que hace necesario mayor educación sobre los componentes de la grasa y su efecto en la salud.

Aunque se identificó que el precio de la carne es un factor que condiciona la percepción del consumidor, el hallazgo de la valoración del bienestar animal como componente de la percepción extrínseca de la carne podría permitir el desarrollo de sellos de carne con énfasis en bienestar animal que aumenten la aceptación de la carne y posibiliten una mayor valoración económica del producto.

Las características del consumidor que más influyeron en el concepto de percepción de la calidad de carne bovina fueron: el género, la edad y la frecuencia de consumo. Esta tipología de consumidor estuvo asociada a conceptos de consumo saludable y contenido de grasa. 


\section{AGRADECIMIENTOS}

Los autores agradecen especialmente al Ministerio de Ciencia y Tecnología de Colombia por el apoyo brindado en la convocatoria de doctorado nacional 727 de 2015; así como también, a la estrategia de sostenibilidad de los grupos de investigación GRICA y Biogénesis de la Universidad de Antioquia, a la Corporación Colombiana de Investigación Agropecuaria (Agrosavia) y a la marca de carne "Quality Beef" de Euro Supermercados.

\section{REFERENCIAS}

[1] HENCHION, M.; MCCARTHY, MARY; RESCONI, VIRGINIA C. Beef quality attributes: A systematic review of consumer perspectives. Meat Science, v. 128, 2017, p. 1-7.

https://doi.org/10.1016/j.meatsci.2017.01.006

[2] GRUNERT, KLAUS G.; BREDAHL, LONE; BRUNS $\varnothing$, KAREN. Consumer perception of meat quality and implications for product development in the meat sector - A review. Meat Science, v. 66, n. 2, 2004, p. 259-272. https://doi.org/10.1016/S0309-1740(03)00130-X

[3] FONTI-FURNOLS, MARIA; GUERRERO, LUIS. Consumer preference, behavior and perception about meat and meat products: An overview. Meat Science, v. 98, n. 3, 2014, p. 361-371. https://doi.org/10.1016/j.meatsci.2014.06.025

[4] JOO, S.T.; KIM, G.D.; HWANG, Y.H.; RYU, Y.C. Control of fresh meat quality through manipulation of muscle fiber characteristics. Meat Science, v. 95, n. 4, 2013, p. 828-836. https://doi.org/10.1016/j.meatsci.2013.04.044

[5] FRANK, D.; OYTAM, Y.; HUGHES, J. Sensory Perceptions and New Consumer Attitudes to Meat. In: New Aspects of Meat Quality. Tandil (Argentina): Elsevier, 2017. p. 667-698. https://doi.org/10.1016/B978-0-08-100593-4.00028-X

[6] DOS SANTOS, DAVID; ESTEVES-DE OLIVEIRA, TAMARA; GIANEZINI, MIGUELANJELO. Consumer's perception on beef sustainability in Porto Alegre, southern Brazil. Business Management Dynamics, v. 7, n. 02, 2013, p. 7-19.

[7] ARDESHIRI, ALI; ROSE, JOHN-MATTHEW. How Australian consumers value intrinsic and extrinsic attributes of beef products. Food Quality Preference, v. 65, 2018, p. 146-163. https://doi.org/10.1016/j.foodqual.2017.10.018

[8] LIANA, MIMI; RADAM, ALIAS; YACOB, MOHD-RUSLI. Consumer perception towards meat safety: Confirmatory factor analysis. International Journal of Economics Management, v. 4, n. 2, 2010, p. 305-318.

[9] VOEGELE, H.; RON, O.S.; GARMYN, A.J.; O'QUINN, T.G.; BROOKS, J.C.; MILLER, M.F. Consumer Perception of Beef Palatability Altered by Brand Recognition. Meat Muscle Biology, v. 1, n. 3, 2017, p. 8. https://doi.org/10.221751/rmc2017.008

[10] FLOWERS, SARAH; MCFADDEN, BRANDON R.; CARR, CHAD C.; MATEESCU, RALUCA G. Consumer preferences for beef with improved nutrient profile. Journal of Animal Science, v. 97, n. 12, 2019, p. 1-15. https://doi.org/10.1093/jas/skz327.

[11] NGAPO, T.M.; BRAÑA-VARELA, D.; RUBIO-LOZANO, M.S. Mexican consumers at the point of meat purchase. Beef choice. Meat Science, v. 134, 2017, p. 34-43. https://doi.org/10.1016/j.meatsci.2017.07.013

[12] TROY, D.J.; KERRY, J.P. Consumer perception and the role of science in the meat industry. Meat Science, v. 86, n. 1, 2010, p. 214-226. https://doi.org/10.1016/j.meatsci.2010.05.009

[13] BANOVIĆ, MARIJA; CHRYSOCHOU, POLYMEROS; GRUNERT, KLAUS G.; ROSA, PEDRO J.; GAMITO, PEDRO. The effect of fat content on visual attention and choice of red meat and differences across gender. Food Quality Preference, v. 52, 2016, p. 42-51. https://doi.org/10.1016/j.foodqual.2016.03.017 
[14] ENCISO, KAREN; BURKART, STEFAN; CHARRY, ANDRES; PUERTA-RODRIGUEZ, CRISTHIAN-DAVID; MUÑOZ, JHON-JAIRO; RUÍZ, ROCIO; GUTIERREZ, JHON-FREDDY; DÍAZ, MANUEL; VIVAS-QUILA, NELSON-JOSE; ALBAN-LOPEZ, NOÉ; MORALES-VELASCO, SANDRA; PETERS, MICHAEL. Consumer Preferences and Market Segmentation for Differentiated Beef with Less Environmental Impact. In Congress Solidarity in a competing world fair use of resources. Viena (Austria): Tropentag, 2016.

[15] FEDERACIÓN COLOMBIANA DE GANADEROS (FEDEGAN). Ganadería Colombiana. Hoja de ruta 20182022. Bogotá (Colombia): Federación Colombiana de Ganaderos, 2018, 126 p.

[16] SANTANA, A.; CAMACHO, C.; ESTÚVEZ, L.; GUTIÉRREZ, J.; GÓMEZ, M.; GARCÍA, G.; BALLESTEROS, $\mathrm{CH} . \mathrm{H}$. Competir e innovar la ruta de la industria bovina :agenda prospectiva de investigación y desarrollo tecnológico para la cadena cárnica bovina en Colombia. Bogotá (Colombia): Ministerio de Agricultura y Desarrollo Rural, 2009, p. 245.

[17] GARCÍA, G.; RUÍZ, D. Plan Estratégico de Ciencia, Tecnología e Innovación del Sector Agropecuario Colombiano. Cadena Cárnica Bovina. Bogotá (Colombia): Agrosavia, 2016, p. 48.

[18] FEDERACIÓN COLOMBIANA DE GANADEROS (FEDEGAN). Así es nuestro consumidor. Bogotá (Colombia): Revista Carta Fedegan, 111, p.22-6.

[19] FRANK, DAMIAN; SEON-TEA, JOO; WARNER, ROBYN. Consumer acceptability of intramuscular fat. Korean Journal Food Science Animal Resource, v. 36, n. 6, 2016, p.609-708. https://doi.org/10.5851/kosfa.2016.36.6.699

[20] BARRAGÁN, WILSON; MAHECHA, LILIANA; ANGULO, JOAQUÍN; OLIVERA, MARTHA. Percepción de consumidores frente a información nutricional de la carne bovina. Idesia, v. 36, n. 4, 2018, p. 35-43. http://dx.doi.org/10.4067/S0718-34292018005002302

[21] MEJÍA-ARANGO, L.J.; ESTRADA, C.M.; SALCEDO-AMAYA, G.J.; HIGUITA, J.M.; GRANADOS, J.D. Informe de calidad de vida Medellìn 2016. In: Medellín Cómo vamos. Medellín (Colombia): 2016.

[22] ALINDA, F.; KAVOI, M.; MIGISHA, J. Consumer willingness to pay for quality beef in Kampala, Uganda. Journal Agricultural Science Technology, v. 17, n. 2, 2017, p. 59-77.

[23] XUE, HONG; MAINVILLE, DENISE; YOU, WEN; NAYGA, J.R.; RODOLFO, M. Consumer preferences and willingness to pay for grass-fed beef: Empirical evidence from in-store experiments. Food Quality Preference, v. 21, n. 7, 2010, p. 857-866. https://doi.org/10.1016/j.foodqual.2010.05.004

[24] HAIR, JOSHEP; BLACK, WILLIAM; BABIN, BARRY; ANDERSON, ROLPH. Multivariate Data Analysis. London (United Kingdom): Pearson, 2017, 734 p.

[25] REVELLE, WILLIAM. psych: Procedures for Psychological, Psychometric, and Personality Research. R package [online]. 2020. Disponible: https://cran.r-project.org/web/packages/psych/psych.pdf [citado 11 de marzo de 2020]

[26] ROSSEEL, YVES. Lavaan: An R package for structural equation modeling and more. Version 0.5-12 (BETA). Journal Statical Software, v. 48, n. 2, 2012, p. 1-36. 10.18637/jss.v048.i02

[27] BEAUJEAN, ALEXANDER A. Latent Variable Modeling Using R: A Step-by-Step Guide. New York (United State): Routledge, 2014, p. 1-205.

[28] COLOMBIA. DEPARTAMENTO ADMINISTRATIVO NACIONAL DE ESTADÍSTICA (DANE). Boletín Técnico. Censo nacional de población y vivienda (CNPV)-2018. [online]. 2019. Disponible en: https://www.dane. gov.co/index.php/estadisticas-por-tema/demografia-y-poblacion/censo-nacional-de-poblacion-y-vivenda-2018 [citado 11 de marzo de 2020]

[29] BAGOZZI, RICHARD P.; YOUJAE, YI. On the Evaluation of Structural Equation Models. Journal of Academy Marketing Science, v. 16, n. 1, 1988, p. 74-94. http://jam.sagepub.com/cgi/doi/10.1177/009207038801600107

[30] MERLINO, V.M.; BORRA, D.; GIRGENTI, V.; DAL VECCHIO, A.; MASSAGLIA, S. Beef meat preferences of consumers from Northwest Italy: Analysis of choice attributes. Meat Science, v. 143, n. 2016, 2018, p.119-128. https://doi.org/10.1016/j.meatsci.2018.04.023 
[31] FLOWERS, S.; MCFADDEN, B.; CARR, C.; MATEESCU, R. Understanding Beef Nutritional Attributes Contributes to Consumers' Willingness-to-Pay for a Healthier Product. Meat Muscle Biololy, v. 2, n. 2, 2018, p. 15-16.

[32] ARENAS-DE MORENO, LILIANA; JEREZ-TIMAURE, NANCY; VALERIO-HERNÁNDEZ, JONTHAN; HUERTA-LEIDENZ, NELSON; RODAS-GONZÁLEZ, ARGENIS. Attitudinal Determinants of Beef Consumption in Venezuela: A Retrospective Survey. Foods, v. 9, n. 202, 2020, p.1-18. https://doi.org/10.3390/foods9020202

[33] KIM, Y.H.B.; MA, D.; SETYABRATA, D.; FAROUK, M.M.; LONERGAN, S.M.; HUFF-LONERGAN, E.; HUNT, M. CUnderstanding postmortem biochemical processes and post-harvest aging factors to develop novel smart-aging strategies. Meat Science, v. 144, 2018, p. 74-90. https://doi.org/10.1016/j.meatsci.2018.04.031

[34] MIRANDA-DE LA LAMA, G.C.; ESTÉVEZ-MORENO, L.X.; SEPULVEDA, W.S.; ESTRADA-CHAVERO, M.C.; RAYAS-AMOR, A.A.; VILLARROEL, M.; MARÍA, G.A. Mexican consumers' perceptions and attitudes towards farm animal welfare and willingness to pay for welfare friendly meat products. Meat Science, $v$. 125, 2016, p. 106-113. https://doi.org/10.1016/j.meatsci.2016.12.001

[35] LUSK, JAYSON L. Consumer beliefs about healthy foods and diets. PLoS One, v. 14, n. 10, 2019, p. 1-15. https://doi.org/10.1371/journal.pone.0223098

[36] SCOLLAN, NIGEL D.; PRICE, ELERI M.; MORGAN, SARAH A.; HUWS, SHARON A.; SHINGFIELD, KEVIN J. Can we improve the nutritional quality of meat?. Proceedings of the Nutrition Society, v. 76, n. 4, 2017, p. 603-618. https://doi.org/10.1017/S0029665117001112

[37] REALINI, C.E.; KALLAS, Z.; PÉREZ, JUAN M.; GÓMEZ, I.; OLLETA, J.L.; BERIAIN, M.J.; SAÑUDO, C. Relative importance of cues underlying Spanish consumers' beef choice and segmentation, and consumer liking of beef enriched with n-3 and CLA fatty acids. Food Quality Prefererence, v. 33, 2014, p. 74-85. https://doi.org/10.1016/j.foodqual.2013.11.007 